\title{
Proyectos sociales deportivos, carreras de deportistas y ex-deportistas y el don entre extraños
}

\author{
Guedes, Simoni Lahud \\ Universidade Federal Fluminense - Conselho Nacional de Desenvolvimento \\ Científico e Tecnológico (CNPq), Brasil \\ simonilahud@uol.com.br
}

Traducción de: Verónica Capasso

Dedicado a Pablo Alabarces, interlocutor y amigo con quien mucho he aprendido

Cita sugerida: Guedes, S. L. (2018). Proyectos sociales deportivos, carreras de deportistas y ex-deportistas y el don entre extraños. Cuestiones de Sociología, 18, e049. https://doi.org/10.24215/23468904e049 


\section{Proyectos sociales deportivos, carreras de deportistas y ex-deportistas y el don entre extraños}

Sports social projects, careers of athletes and ex-athletes and the gift between strangers

Simoni Labud Guedes

Universidade Federal Fluminense - Conselho Nacional de

Desenvolvimento Cientifico e Tecnológico (CNPq), Brasil

simonilahud@uol.com.br

\section{Resumen:}

En este artículo propongo analizar los cambios ocurridos desde 1994 en las representaciones colectivas sobre la selección brasileña de fútbol. Argumento que el aumento extraordinario de la mercantilización del fútbol profesional, en especial en Europa, transformó a los jugadores en multimillonarios que residen en el exterior, condición que dificulta la relación metonímica establecida, anteriormente, entre la selección nacional brasileña y el pueblo de Brasil. Establezco una conexión entre esas representaciones y el gran número de proyectos sociales deportivos emprendidos en Brasil por atletas de alto rendimiento, entre ellos jugadores que fueron parte de la selección brasileña de fútbol en las Copas del Mundo de la Federación Internacional de Fútbol Asociado (FIFA), en especial a partir de 1994. Estos datos son interpretados a partir de la teoría del don de Marcel Mauss. Además, se realizó una comparación con datos argentinos sobre proyectos sociales deportivos que arrojó luz sobre el caso brasileño. Palabras ClaVe: Proyectos sociales deportivos, Selección brasileña, Futbol, Teoría del don, Argentina.

\section{Abstract:}

In this text, I discuss the changes in the collective representations of the Brazilian football team since 1994. I argue that the extraordinary increase in the commercialization of professional football, particulary in Europe, turned these players into multimillionaires residing abroad, which hampers the previously established metonymic relationship between Brazilian football team and Brazilian people. I make a connection between these representations and the large number of social sports projects undertaken in Brazil by high-performance athletes, among them players who played in the Brazilian soccer team at the FIFA World Cups, especially since 1994. These data are interpreted according to Marcel Mauss'theory of the gift. In addition, there was a comparison to Argentine data on social sports projects that, by contrast, shed light on Brazilian material.

KEYWORDS: Social sports projects, Brazilian Team, Football, Theory of gift, Argentina.

\section{ENCRUCIJADAS EN UNA TRAYECTORIA DE INVESTIGACIÓN}

\subsection{La selección nacional de fútbol y el pueblo brasileño}

Desde la década de 1970 (cf. Guedes, 1977) vengo trabajando con dos líneas de investigación simultáneas. Por un lado, lo que más tarde sería rotulado como antropología de las prácticas deportivas y, por otro, la antropología de las clases trabajadoras o, más comúnmente, de las clases populares. Conjugué estas dos líneas en mi primer trabajo académico, la tesis de maestría, referenciada anteriormente. En una primera parte, realicé estudios sobre el fútbol brasileño, con foco en la selección brasileña en las Copas del Mundo. En una segunda parte, expuse los resultados de una etnografía realizada, por más de un año, con obreros de una fábrica de tejidos -en ese entonces existente- en el barrio de Bangu, zona oeste de la ciudad de Río de Janeiro, conocida como Fábrica de Tejidos Bangu (Compañía Progreso Industrial de Brasil, fundada en 1889). Allí, focalicé en sus representaciones y prácticas ligadas al fútbol.

En ese artículo ${ }^{1}$, entonces, investigué, inicialmente, las representaciones sobre Brasil y los brasileños presentes en tres periódicos de gran circulación en Río de Janeiro y en el país ${ }^{2}$ ( $O$ Globo, O Dia e Jornal 
dos Sports), siguiendo las noticias, crónicas y reportajes sobre la Copa del Mundo de 1950 y la Copa del Mundo de 1970. En la segunda parte del trabajo, presenté los sentidos y significado del fútbol, así como su práctica amateur -y todo lo que lo rodea- en la vida de obreros de la Fábrica Bangu con los cuales conviví en la etnografía.

Pues bien, desde ese momento, me quedó claro que el desempeño de la selección brasileña de fútbol en las Copas del Mundo, al menos a partir de 1950 -la IV Copa del Mundo FIFA ${ }^{3}$ - era interpretado como resultado de las cualidades y defectos del pueblo brasileño. Es decir, desde entonces, distinguiendo elites y pueblo, los jugadores de fútbol eran vistos como representantes del pueblo, en una ecuación bastante directa, casi sin mediaciones. En ese sentido, los jugadores de fútbol serían provenientes de las capas populares y su desempeño, para bien o para mal, reproduciría las potencialidades y limitaciones de estas capas. De este modo, selección brasileña $=$ pueblo brasileño, tanto en el sentido metafórico como metonímico. Todas las narrativas sobre la selección, entonces, podrían ser interpretadas como narrativas sobre el pueblo. Era un determinado Brasil el que se pretendía leer en los campos de fútbol.

De cierta forma, en la segunda parte de la tesis de maestría, la etnografía con los obreros de la Fábrica Bangu confirmaba la extensión de esta interpretación. Presentaban una visión crítica de todo el fútbol ${ }^{4}$ y también consideraban que este rendimiento, con tres victorias resonantes en este periodo (1958, 1962 y 1970), fue el resultado de la habilidad y el arte de las clases bajas, en un claro rechazo del intento de apropiarse de la victoria de 1970 por la dictadura militar, entonces presidida por el general Emilio Garrastazu Médici (1969-1974).

Continué trabajando en esta clave de interpretación analítica, la del desempeño de Brasil en la Copa Mundial -ideología de la victoria e ideología de la derrota, la ecuación entre la selección brasileña y el pueblo brasileño-, en las siguientes décadas en las que el equipo recogió derrotas (Copas de 1974, 1978, 1982, 1986, 1990). El seleccionado vuelve a ser victorioso en 1994, en Estados Unidos, en un desempeño que, hasta hoy, se considera al menos atípico. Esta, tal vez, sea la victoria menos recordada del fútbol brasileño.

Sin embargo, un fenómeno social decisivo ocurrió a partir de 1990: la venta de los jugadores más valorados en el mercado futbolístico para clubes en el exterior, la mayoría clubes europeos. Las últimas décadas del siglo $\mathrm{XX}$ han visto un crecimiento asombroso del mercado deportivo, en el cual las principales mercancías son los jugadores. Brasil, junto con otros países de la periferia capitalista, se convirtió en un exportador de cracks. Esta simple tabla lo demuestra con claridad:

Jugadores convocados brasileños para las Copas del Mundo (1986-1998)

\begin{tabular}{|l|l|l|}
\hline Número de convocados & Jugando en Brasil & Jugando en el exterior \\
\hline $1986-23$ convocados & 21 & $2^{5}$ \\
\hline $1990-22$ convocados & 10 & $12^{6}$ \\
\hline $1994-22$ convocados & 12 & $10^{7}$ \\
\hline $1998-22$ convocados & 08 & $14^{8}$ \\
\hline
\end{tabular}

Si consideramos al equipo titular de $1998^{9}$, con una u otra sustitución, en realidad sólo tendríamos jugando en Brasil el arquero Taffarel y al defensor Júnior Baiano. Todos los otros 9 titulares jugaban en el exterior (véase la nota 8 ).

Estos pocos datos son síntomas de un fenómeno avasallador: la mercantilización del fútbol profesional. Claro que desde sus inicios, en el siglo XIX, hubo mercantilización del fútbol. Sin embargo, lo que ocurrió a partir de 1990 es un fenómeno sin precedentes, cuyo centro fue Europa (cf. Alabarces, 2014):

Desde la Copa del Mundo de 1990, en Italia, la industria del fútbol europeo entró en una fase diferente, la posmoderna. La renta de la televisión revolucionó la economía política del juego, permitiendo que los clubes buscaran nuevos y más ricos aficionados para sentarse en el cómodo estadio reconstruido. (...) era tendencia de los productores de artículos deportivos 
contratar al jugador para los comerciales de sus mercancías, a menudo en detrimento de los compromisos vinculados a los negocios del club (Giulianotti, 2002, p. 137) ${ }^{10}$.

Las dimensiones de esta supermercantilización de finales del siglo XX son innumerables. Para mi argumento, destaco la intensificación y ampliación de la venta de jugadores de los países periféricos, especialmente América Latina y África, para los grandes clubes europeos, principalmente, pero también para clubes de otros países. De la misma manera, los principales jugadores son comprados por cifras inimaginables y reciben, además de altísimos salarios, derechos de imagen, contratos publicitarios millonarios, etc. O sea, se convierten en multimillonarios, a veces de la noche a la mañana.

En el caso brasileño, este movimiento asumió diversos significados. Terminó la era en que jugadores como Pelé o Zico permanecieron prácticamente toda su vida profesional en el club en el cual se estrenaron. Se hizo imposible, desde entonces, mantener a un joven muy prometedor en un equipo nacional, pues las transferencias pasaron a llevarse a cabo cada vez más temprano ${ }^{11}$.

Desde el punto de vista simbólico, esta dimensión de la mercantilización del fútbol tiene varias implicaciones:

a) Como se ve en el equipo titular brasileño de la Copa del Mundo de 1998, sólo 2 jugadores aún jugaban en Brasil. En esta Copa, los medios empiezan a llamarlos europeos o extranjeros (Guedes, 2003 , donde traté más largamente sobre esta clasificación ${ }^{12}{ }^{13}$ ), lo que propicia un oxímoron: se convierten en los héroes nacionales extranjeros.

b) Los jugadores de fútbol seleccionables de esta fase posmoderna, como dice Giulianotti (2002), se convirtieron en multimillonarios, con sus vidas expuestas constantemente en los medios de comunicación.

De este modo, la identificación de la selección brasileña de fútbol con el pueblo brasileño, a través de sus jugadores, deja de ser metonímica aunque, en algunas circunstancias, sigue siendo metafórica. Ejemplificando esta operación metonímica, en su auge, tenemos la valoración de João Lyra Filho (1906-1988), jefe de la delegación brasileña de la Copa del Mundo de 1954, en Suiza, tras la cual publicó su informe explicando un nuevo fracaso:

El estado psicosocial de nuestro pueblo todavía enverdece y los atletas salidos del medio del pueblo no pueden improvisar condiciones e instrumentos de superación ante aquellas pruebas deportivas que exigen la movilización de mayores recursos y reservas orgánicas (Lyra Filho, 1954, p. 52). ${ }^{1415}$

João Lyra Filho deja bastante clara la distinción entre una élite educada, a la que él y los dirigentes pertenecen, y el pueblo genética y socialmente empobrecido.

Quedémonos aquí por ahora, habiendo establecido que la ecuación selección brasileña de fútbol = pueblo brasileño, vigente desde, por lo menos, 1938, sufrió algunos estremecimientos a partir del final del siglo XX. En este período, aunque buena parte de los seleccionables sigue siendo oriunda de las capas más pobres de la población, al convertirse en jugadores de éxito se enriquecen y muchos se van a residir en el exterior.

Pasemos a otras investigaciones que he venido realizando más recientemente para demostrar el hecho de que otros caminos nos conducen de vuelta al seleccionado brasileño en las Copas del Mundo.

\subsection{Proyectos sociales deportivos ${ }^{16}$}

En 2003, inicié un nuevo proyecto de investigación denominado "Socialización y profesionalización a través de los deportes en los proyectos sociales deportivos". Este proyecto continuaba la investigación que venía haciendo con jóvenes de clase trabajadora, particularmente con relación a la iniciación al trabajo (Guedes, 1997). El objetivo principal de la investigación era analizar la forma en que los proyectos sociales deportivos 
eran apropiados por estos jóvenes, ya que estos proyectos se posicionaban como complemento educativo, siendo que algunos ofrecían cursos para la profesionalización. También buscaba comprender la enorme y visible proliferación de estos proyectos en todo Brasil.

Comenzamos, algunos becarios de Iniciación Científica y yo, por mapear los principales proyectos sociales deportivos en la ciudad de Niterói, región metropolitana de Río de Janeiro, ciudad en la que se sitúa la sede de la Universidad Federal Fluminense en la que estamos insertos. En Niterói hay un gran proyecto denominado "Nombres", parcialmente una política pública del Ayuntamiento Municipal de Niterói, que proporciona algunos subsidios para su realización, entre ellos espacio urbano, profesionales de apoyo, etc. El proyecto "Nombres" pretende atender a niños y jóvenes de baja renta ${ }^{17}$, privilegiando a los estudiantes de la escuela pública. Incorpora, a través de sus subsidios, cuatro institutos orientados a los niños y uno hacia las personas mayores. En la continuidad del trabajo de investigación, percibimos que los proyectos tienen propósitos muy similares. Ejemplifico a continuación con los propósitos (misión es la categoría nativa utilizada) de aquellos insertados en el proyecto "Nombres". Son ellos:

a) Instituto Canhotinha de Ouro, epíteto del jugador Gérson, uno de los titulares de la selección brasileña de fútbol de 1970, que a través del fútbol se propone auxiliar en el acompañamiento escolar, crear hábitos saludables de práctica deportiva, socializar e integrar a los niños, auxiliar en la alimentación a través de complementación alimentaria suministrada en los diversos núcleos existentes, prevenir enfermedades a través de los convenios médicos y odontológicos (cf https://ww w.facebook.com/pg/InstitutoCanhotinhadeOuro, acceso el 8 de mayo de 2018).

b) Instituto Fernanda Keller, triatleta, que "tiene como misión la Educación Social, a través del deporte, promoviendo la socialización e integración social de niños y jóvenes sobre la base de las acciones integradas que involucran deporte, salud, ocio y entretenimiento" (cf. http://www.institutofernand akeller.com.br/instituto-fernanda-keller, acceso el 8 de mayo de 2018).

c) Instituto Rumo Náutico e Projeto Grael, ideado por los hermanos Torben y Lars Grael, campeones olímpicos de vela. Este Instituto, además de iniciar a los jóvenes de escuelas públicas en la vela, ofrecen cursos para trabajar con profesiones ligadas a la vela. Tienen un núcleo en la ciudad de 3 Marías, Minas Gerais (Cf. http://www.projetograel.org.br/institucional/organograma/index.php, acceso el 8 de mayo de 2018).

d) Proyecto Tatuí enseña surf y bobyboarding, pero principalmente invierte en la educación ambiental, con foco en la limpieza de los mares (No tiene sitio web).

Además de estos cuatro proyectos dirigidos a niños y adolescentes de capas populares, el proyecto Nombres del Municipio de Niterói aún alberga el Proyecto Gugu (FUNCAB - Fundación Profesor Carlos Augusto Bittencourt), con más de treinta núcleos en Niterói, ofreciendo gimnasia para ancianos en espacios públicos (véase http://www.funcab.org/gugu.php, acceso el 8 de mayo de 2018).

Los tres primeros proyectos fueron objeto de etnografías de mis becarios de graduación en Ciencias Sociales (cf. Novaes, 2006; Davies, 2006; Oliveira Filho, 2005; Guedes, Davies y Novaes, 2006). Sin embargo, además de los trabajos etnográficos, que nos trajeron informaciones importantísimas, yo tenía intención de hacer un mapeamiento de los proyectos sociales deportivos, al menos en la región metropolitana del gran Río de Janeiro. Uno de mis becarios, Robson Campaneruti da Silva (2007), se encargó de este trabajo, debiendo visitar instituciones que tuvieran registros de estos proyectos, buscando también en Internet la información adecuada.

El trabajo de Robson Campaneruti nos dejó, inicialmente, una enorme frustración: rápidamente descubrimos que sería imposible mapear sistemáticamente todos los proyectos sociales deportivos en la región metropolitana de Río de Janeiro. Hay proyectos grandes, pequeños, medios, la mayoría sin registro en ningún lugar. Cada tanto éramos informados de algún proyecto por los medios, por otros investigadores. Al mismo 
tiempo, percibimos la rapidez con que surgían y desaparecían: descubríamos un proyecto en internet y, al buscarlo, éramos informados de que había desaparecido.

Sin embargo, este trabajo también abrió una línea de investigación mucho más importante. El levantamiento del proyecto en internet demostró algunos aspectos muy relevantes y que condujeron mis investigaciones posteriores. En primer lugar, era extraordinario el número de proyectos sociales deportivos que fueron idealizados por atletas en actuación y con carreras encerradas, siendo la mayoría jugadores de fútbol seleccionables; en segundo lugar, muchos de estos proyectos llevan el nombre del atleta o su epíteto (por ejemplo, el ya citado Canhotinha de Ouro); en tercer lugar, además de cada uno dedicarse al deporte en el que se consagró, realizaban, en general, el proyecto social deportivo en su lugar de origen o en el lugar en que comenzaron la práctica deportiva exitosa. Finalmente, observamos que la gran mayoría de estos proyectos sociales deportivos emprendidos por jugadores de fútbol tuvo su inicio a partir de 1994, en un movimiento que se intensificó de forma extraordinaria en los años siguientes.

En 2006, y posteriormente en 2009, elaboré un proyecto comparativo para verificar si en Argentina, país exportador de cracks, había la misma proliferación de proyectos sociales deportivos realizados por jugadores de fútbol exitosos. Existe en Argentina un número muy grande de ONGs, tal como en Brasil. Además, en la década de 1990, se estimaba más de tres mil organizaciones en todo el país y más de la mitad dirigidas a niños y adolescentes (véase Filmus, 1997).

Comencé a desarrollar el trabajo de campo con la expectativa de que, al menos en el Gran Buenos Aires, encontraría muchos proyectos sociales emprendidos por los innumerables jugadores de fútbol de éxito internacional, millonarios y trabajando en los grandes clubes europeos. Sin embargo, esta expectativa fue frustrada. De un lado, porque entre las innumerables ONGs en actuación dirigidas a niños y jóvenes, pocas están centradas en los deportes como estrategia pedagógica. Por otro lado, en 2006 había simplemente una única fundación emprendida por un jugador: la Fundación Pupi de Javier Zanetti. En 2007 surge la Fundación Leonel Messi, con sus tres pilares: salud-educación-deporte (véase http://www.fundacionleomes si.org), consecuencia de acciones aisladas que realizaba anteriormente, financiando tratamientos de niños, en especial aquellos de crecimiento, similares a los que él mismo había vivido. No pude comprobar en relación a la fundación de Messi, pero sí que la fundación Pupi recibía donaciones de jugadores de éxito de varios deportes, como el astro del tenis David Nalbandian. Sin embargo, proyectos sociales al estilo brasileño, apenas había uno en 2006, con la creación de otro en 2007. Como se ve, la situación era absolutamente opuesta con el caso brasileño, a diferencia de mis expectativas.

La Fundación Pupi, que he visitado algunas veces, tiene explícitamente, según su director Andrés de La Fuente, suegro del jugador, inspiración en el modelo brasileño, específicamente en la Fundación Gol de Letra, de los jugadores Leonardo y Raí. De La Fuente afirma que la relación personal de Javier Zanetti y Leonardo fue esencial para el emprendimiento. La fundación fue creada en 2002, luego de la gran crisis económica / de 2001. El discurso legitimador de este emprendimiento es muy semejante a los discursos de los proyectos sociales deportivos brasileños. Sigo, a través de los comunicados que periódicamente recibo, el crecimiento de esta fundación, la ampliación de los servicios que ofrece, incluso con varios programas de atención a las familias de los niños y adolescentes atendidos.

\section{INTERMEDIO: UN ARGUMENTO}

Estos dos diferentes programas de investigación -los estudios sobre selección brasileña en las Copas del Mundo y los estudios sobre proyectos sociales deportivos- confluyeron en una cuestión. ¿Por qué, en Brasil, a partir de 1994, prácticamente todos los jugadores que fueron seleccionados para Copas del Mundo dieron sus nombres y sus esfuerzos para crear y mantener proyectos sociales deportivos? Mi argumento es que eso se transformó en una obligación, dentro de un circuito amplio del don (Mauss, (1968 [1923-1924]) que 
implica el desempeño de la selección brasileña en la Copa Mundial. Eso es lo que vamos a ver a continuación, en la segunda parte de este artículo, revisitando el texto seminal de Marcel Mauss.

\section{El gRAN CIRCUITO DEL DON ENTRE LOS JUGADORES DE FÚTBOL}

\subsection{El Essai sur Le don}

Más de ochenta años después de su publicación, el Essai sur Le don ${ }^{18}$ continua en el centro de algunos debates teóricos, en especial en la antropología, y, principalmente, continúa inspirando una serie de apropiaciones analíticas. No es necesario, en mi opinión, discutir aquí los innumerables rodeos teóricos de las diferentes interpretaciones de la herencia de Mauss, la cual propició distintas lecturas de su propuesta analítica. Pero me parece necesario apuntar aquí la forma en que estoy interpretando los puntos básicos de este texto para que algunas cuestiones puedan ser discutidas a continuación. Como sabemos, dos nociones fundamentales han sido objeto de discusión: la noción de hecho social total y el principio del intercambio (prestaciones) como elemento fundante de la sociedad, denominado posteriormente por Lévi-Strauss, de principio de la reciprocidad. Este principio se resume en la triple obligación: dar, recibir, retribuir. Esta fórmula, en su aparente simplicidad, cubre algunas de las cuestiones más complejas de las ciencias sociales y fue a menudo interpretada como la creación del eslabón fundador que posibilita la vida social.

En esta obra, como fue señalado por diversos comentaristas, Mauss recrea algunos de los temas durkheimianos básicos, posicionándose claramente dentro del legado de la Escuela Sociológica Francesa pero, ciertamente, superando algunas de sus limitaciones. En especial, sobrepasa el llamado objetivismo durkheimiano (cf. Bourdieu, 1972), buscando en el movimiento de la sociedad, en el movimiento y la actuación de los sujetos, vistos simultáneamente como productos y productores de su sociedad: "El dato es Roma, es Atenas, es el francés de clase media, es el melanésio de esa o aquella isla, y no la oración o el derecho en sí" (Mauss, 2003, p. 311). En este punto de vista, Mauss, en cierto modo, inaugura lo que Philippe Corcuff (2001) va a denominar una "problemática constructivista" en la que, evidentemente, se sitúa también la noción de habitus tal como fue elaborada en varios textos por Bourdieu (por ejemplo, 1972). Para Allan Caillé, más que eso, estaríamos frente a lo que él llama el tercer paradigma -el paradigma del don y del simbolismo-, único capaz de superar las limitaciones, por un lado, del holismo y, por otro lado, del individualismo metodológico. En cierto punto de vista, en las palabras de este autor:

A partir de Durkheim, la Sociología francesa sólo se realiza plenamente con Mauss, a partir del momento en que éste consigue reformular las cuestiones planteadas por su tío en el único campo en que son pasibles de ser respondidas, el de la naturaleza de lo simbólico y de su vinculación con la obligación de dar. (Caillé, 1998) ${ }^{19}$

En este artículo tomo el principio de la reciprocidad como instrumental analítico para interpretar los datos presentados en la primera parte de este texto. Hago una propuesta interpretativa acerca de la proliferación de proyectos sociales deportivos emprendidos por jugadores exitosos en Brasil, contrastando con su casi inexistencia en Argentina. Espero demostrar también algunos aspectos de la forma en que, en cada una de estas sociedades - la brasileña y argentina-, la urdimbre entre las clases y segmentos sociales está representada. De paso, señalo que pienso que este análisis también permite comprender algunas de las dimensiones de la producción de las identidades nacionales, tema que he abordado en otras oportunidades.

Para lo que pretendo demostrar, considero especialmente fecundo el desdoblamiento propuesto por Jacques Godbout, texto con la colaboración de Allan Caillé (1999), formulando la hipótesis de que "el don no sólo afecta a las sociedades primitivas, sino también, aunque de una manera alterada, que aún debe analizarse, a la sociedad contemporánea" (p. 28, las cursivas son mías). Esta propuesta conduce al análisis de intercambios navideños, herencia, donación de sangre y de órganos, grupos de ayuda mutua, etc. Abre todavía 
un extenso campo empírico para la reflexión sobre el regalo en las sociedades modernas. También demuestra la irreductibilidad de los circuitos de reciprocidad tanto a la lógica del mercado cuanto a la intervención estatal, aunque sea casi imposible, en la actualidad, no tener en cuenta el mercado y el Estado.

Los circuitos de reciprocidad orientados por el principio del don en las sociedades contemporáneas deben ser pensados como entrelazados a la economía monetaria capitalista mundializada y a la organización de los Estados nacionales. En este caso, el "enigma del don", como lo nombra Maurice Godelier (2001), hace más compleja la articulación simultánea de obligación y la acción voluntaria, interés y desinterés. En passant, vale la pena señalar un tema relacionado, que es la llamada responsabilidad social corporativa. Es sabido que ninguna empresa, en el mundo contemporáneo, puede extender sus mercados sin presentar esta cara de responsabilidad social.

Este entrelazamiento entre donación, mercado y Estado también genera un expresivo fenómeno de la modernidad que son las llamadas ONGs, organizaciones no gubernamentales que, en el propio epíteto con que se consagran, registran su relación con el Estado. En el caso brasileño, como ya he dicho anteriormente, no hay posibilidad de obtener registros precisos sobre tales organizaciones. Se trata de un campo de actuación dinámico y movedizo, en el que hay una relativa facilidad de crear asociaciones, más o menos reguladas por instrumentos legales estatales. Una gran parte, sin embargo, tiene vida corta, dependiente de financiamientos que, al agotarse, llevan también al cierre de las actividades. Un tema, sin duda relacionado -y en el orden del día en el caso brasileño-, es el vínculo entre ONGs y corrupción, en la medida en que muchos recursos estatales han sido distribuidos para ser gestionados por estas organizaciones constituidas con relativa facilidad. La multiplicación de estas organizaciones - cerca de 30.000 registradas con el gobierno federal (ver Melo, 2005)- ocurre, en su mayor parte (90\%), a partir de la década de 1990.

\subsection{Don recibido, don que tiene que circular}

En Brasil, un número no despreciable de estas ONGs considera que los deportes son inherentemente socializadores y pedagógicos, de modo que se multiplican los proyectos sociales deportivos que tienen por objetivo la inclusión social de niños y jóvenes de las capas más pobres de la población ${ }^{20}$, propuestos, en principio, como donación voluntaria y desinteresada. Estos proyectos se realizan, por un lado, por políticas públicas - como el Programa Segundo Tiempo del Ministerio de Deportes ${ }^{21}$ - o por ONGs. Cabe señalar que, por un lado, estas políticas públicas mantienen estrechas relaciones con el campo de las ONGs, pues gran parte de los recursos públicos son distribuidos, en edictos, para las organizaciones sociales privadas. Evidentemente, esto ha generado numerosos problemas de malversación de fondos. Por otro lado, muchas de estas organizaciones, aunque no todas, sólo existen en función de estos recursos públicos.

Pues bien, cuando empecé la investigación sobre tales proyectos sociales deportivos, hace algunos años, una observación se repetía: era un campo en que la actuación efectiva o el uso del nombre y de la imagen de atletas consagrados era muy frecuente. Involucraba tanto a atletas en actividad como a aquellos que ya habían cerrado sus carreras, como pude comprobar en relevamientos hechos dentro de mi grupo de investigación.

Por un lado, no había nada sorprendente en este fenómeno. Como ya mencioné en la primera parte de este artículo, la explosión del mercado deportivo - para el caso brasileño, en especial del fútbol- en las últimas décadas del siglo XX, ha llevado al enorme enriquecimiento de muchos atletas, extremadamente valorados en este mercado. Y la filantropía o - más modernamente- la contribución a causas sociales no es una novedad en la relación entre clases y segmentos sociales. Lo extraordinario, para mí, era la constancia de un tipo de inversión social específica, podríamos tal vez decir, un "estilo" de donación en que la persona del donante va, necesariamente, junto con la donación. Son innumerables, en Brasil, las organizaciones que llevan el nombre o el "apodo" del atleta donante ${ }^{22}$. Esta característica implica, a menudo, la inversión personal del atleta en el proyecto social deportivo, actuando junto a los niños y adolescentes, emprendiendo 
esfuerzos para la obtención de financiamientos públicos y privados. Cada uno de ellos se presenta como modelo para los jóvenes. Esta característica, en principio, representaba un diferencial en relación a lo que se sabe acerca de las donaciones de los ricos y famosos que, en general, se limitan a la donación de fondos y sólo eventualmente donan su tiempo y su trabajo en presentaciones de beneficencia ${ }^{23}$. Invertir personalmente en un emprendimiento laborioso a largo plazo y que coloca, todo el tiempo, su nombre y su prestigio en juego, aparecía, a mi modo de ver, como una especificidad a ser explicada, dada, incluso, su extensión ${ }^{24}$.

La cuestión, entonces, era comprender este "estilo personalizado" de donación, ciertamente específico dentro de lo que Godbout apunta como la "donación entre extraños" que clasifica como específicamente moderna ${ }^{25}$. ¿Por qué, en el caso brasileño, los atletas y exatletas asumen personalmente este proceso? ¿Por qué no donan simplemente sus recursos y su capital simbólico para que los "profesionales del don" (Godbout, 1999, p. 95) realicen el proceso como suele ocurrir con la mayoría de los artistas y atletas de fama mundial?

Un indicio importante para comprender esta cuestión, desde mi punto de vista, está en la categoría nativa don. Eline Deccache-Maia (2003), en su tesis doctoral, se da cuenta de una importante conexión entre la representación del don y de su obligación de circular, estableciendo así una conexión entre el concepto de posesión de algo mágico, especial e intangible -eldon- y los circuitos de reciprocidad. En el amplio desarrollo de esta cuestión, en el marco de la reflexión sobre la formación de futbolistas en Brasil y Francia, Arlei Sander Damo avanza y sostiene: "El don no es sustancia, sino representación”. Y registrando una serie de expresiones nativas indica su representación como "donación divina", como lo inexplicable, el residuo, el significado que permanece oculto (Damo, 2007, p. 199).

Esta concepción es fundamental para la comprensión de la donación expresada por la inversión en proyectos sociales deportivos: se trata, en realidad, de un contra-don que, en el caso brasileño, debe llevar también la persona del receptor de la "donación divina" con la que fue agraciado. Examinando las características más amplias de estos proyectos sociales, cuya "misión" es claramente interpretada como retribución, aparecen otras características que se destacan.

Como era de esperar, la gran mayoría de los proyectos tiene como actividad principal o foco de la atracción de niños y adolescentes el deporte en el cual el atleta se destacó o se destaca. Más importante aún es el hecho de que casi todos los proyectos se llevan a cabo en el lugar del origen real o simbólico del atleta. De cierta forma, es como si la devolución debiera ser realizada en el punto físico en que se originó, devolviendo a una entidad divina los bienes recibidos. Por ejemplo, devolviendo beneficencia a los niños pobres del lugar en que un específico niño pobre fue agraciado con un don que lo hizo rico y famoso.

Bajo esta perspectiva, es importante pensar en el tiempo como fundamental para la comprensión de este proceso. No se trata de reducir la donación a su dimensión interesada, como parece hacer Bourdieu cuando afirma que:

Es el intervalo temporal entre el don y el contra-don que permite ocultar la contradicción entre la verdad vivida (o deseada) del don como acto generoso, gratuito y sin retribución, y la verdad que el modelo revela, aquella que hace del don un momento de una relación de cambio trascendente a los actos singulares de intercambio(Bourdieu, 1996, pp. 7-8) ${ }^{26}$.

Sin dejar de lado, como parece hacer Bourdieu, la ambigüedad inherente al don -interés y desinterés, obligación y libertad-, pensar el intervalo entre don y contra-don permite pensar un amplio circuito en el que, como diría Mauss, una serie de personas morales se involucran en relaciones de reciprocidad. Como afirma Godbout, lo que importa, en la donación, es su valor de vínculo.

En esta perspectiva, los proyectos sociales deportivos emprendidos personalmente por atletas brasileños forman parte de un gran circuito de la donación que, a diferencia de la mayoría de los circuitos tradicionales que no se sabe dónde empiezan, tiene su punto de origen en un don recibido por una persona que, a partir de ahí, debe, necesariamente hacerlo circular, reinventando continuamente la asimetría característica de todo el proceso; o sea, todo movimiento es, al mismo tiempo, dependiendo de la perspectiva, un dar, un recibir, un retribuir (cf. Godbout, 1999). 
¿Y por qué esta obligación de donarse, devolviendo el don primordial (talento excepcional innato), alcanza tan fuertemente a los atletas brasileños, en especial a los jugadores de fútbol de nivel internacional y de la selección nacional, a partir de la década de 1990? Exactamente porque en este período se enriquecieron de forma espectacular y pasaron a residir en el exterior, siendo hasta apodados, en determinado momento, extranjeros por los medios. Ya no es tan simple considerarlos como parte del pueblo brasileño, tal como ocurría anteriormente.

Esta ruptura en la ecuación selección brasileña de fútbol = pueblo brasileño, que los hacía inmediatos representantes del pueblo, en sus cualidades y defectos, puede ser bien comprendida en las reacciones a la derrota brasileña en la Copa del Mundo de 1998 en Francia. Este juego final estuvo marcado por lo que se conoció como el drama de Ronaldinho (Ronaldo Nazario). En aquel momento, la estrella mayor de la selección brasileña tuvo convulsiones pocas horas antes de la final pero, aun así, fue utilizado por el técnico Zagallo. Como para los brasileños la selección de fútbol sólo pierde para sí misma, en las interpretaciones de la derrota pocos consideraron la superioridad del equipo francés. En cambio, dos interpretaciones dominaron el escenario post-copa: 1) Ronaldinho no debería haber jugado; la comisión técnica sólo lo usó porque fue presionada por la multinacional que lo financiaba; 2) los jugadores de la selección brasileña, muy ricos y viviendo en el exterior, ya no comprendían el significado del fútbol para los brasileños, no se preocupaban por las personas pobres del país y, por lo tanto, no se dedicaban debidamente a obtener la victoria. Sobre estos dos puntos, escribí un artículo hace algunos años (Guedes, 2006, en español 2014a).

\section{CONSIDERACIONES FINALES}

Considero que la multiplicación de los proyectos sociales deportivos hechos por jugadores y ex jugadores es una forma de responder a las acusaciones mencionadas. El hecho de que el enriquecimiento sin límites de los jugadores sea coetáneo de la creación de estos proyectos sociales no es coincidencia, ni casualidad. Es, en mi opinión, la respuesta en una forma física efectiva, reafirmando su conexión con el pueblo brasileño. Tampoco es casual que hagan tales proyectos en sus lugares de origen, reafirmando no haber olvidado que fueron pobres o, al menos, no tan ricos. Un potente signo de esta respuesta, a la que me he referido muchas veces, es la imagen del capitán de la selección brasileña de 2002 en el momento de recibir el trofeo, con una inscripción en su camiseta: "100\% Jardim Irene". Esta expresión, enigmática para muchos, incluso para mí, es fácilmente comprensible dentro de la interpretación que ahora propongo. En el momento crucial de su carrera, visto por miles de millones de personas, Cafú reafirma que no se olvida de su lugar de origen (Jardim Irene, periferia paulista) donde, siguiendo la regla no explicitada de la obligación de dar (retribuir), creó un gran proyecto social para los niños pobres. Más claro que eso, imposible.

Quiero señalar también que este formato de donación de la persona que originalmente recibió el don, o sea, los proyectos sociales deportivos, se inscribe como un fenómeno muy extendido en la sociedad brasileña: lo que he llamado la "misión civilizadora" de elites en relación a las capas más pobres de la población. En Brasil hay una relación entre las clases sociales extremadamente jerárquica, pero también personalizada, modelo en que una igualdad jurídica no se realiza en las relaciones entre clases sociales (cf. DaMatta, 1979; Kant de Lima, 2008). Pretenden, así, intervenir privada y directamente en la cuestión social, produciendo nuevos sujetos, como intenté demostrar en otras ocasiones.

¿Será por esta razón que el caso argentino parece tan diferente del brasileño? Si hay, proporcionalmente, tantas ONGs en Argentina como en Brasil, además de atletas igualmente ricos o, incluso, mejor remunerados, ¿por qué no se donan del mismo modo?

Sin embargo, es interesante que sea muy limitado, como ya demostré, en el caso argentino, lo que estoy denominando como "estilo de donación" en el que la persona del donante va junto con la donación, o mejor, es la donación. Con excepción de la Fundación Pupi de Javier Zanetti, rarísimas organizaciones se constituyen dentro de este modelo ${ }^{27}$. 
En este sentido, se puede especular si, en el caso argentino, las relaciones de donación entre diferentes clases y segmentos sociales no serían, de hecho, más "modernas", más despersonalizadas y, en cierto modo, más igualitarias. Aunque, como afirma O'Donnel, "una sociedad pueda ser, al mismo tiempo, relativamente igualitaria y autoritaria y violenta" (2004, p. 167).

De esta forma, dones y contra-dones, dar-recibir-retribuir, involucrando a muchos atletas también enriquecidos, deben transitar, en esta sociedad que se piensa como más igualitaria, por caminos más despersonalizados.

En este punto, se puede especular también si, a diferencia de la tradición brasileña en que se naturalizó la "misión civilizatoria" de las élites en relación al "pueblo", el vector civilizatorio, en el caso argentino, no sería generado por una tradición más fuerte de participación de las "masas" en el proceso político y económico. Así, el circuito de la donación debe recorrer otros caminos, tal vez más institucionalizados.

Me gustaría terminar con la propuesta de O'Donnel (2004) de explorar "los caminos para la democracia de estos países nuestros, tan parecidos y-como aquí recalco- tan diferentes”.

\section{REFERENCIAS BIBLIOGRÁfiCAS}

Alabarces, P. (2014). Identidad, divino tesoro. En Héroes, machos y patriotas. El fútbol, entre la violencia y los medios. Buenos Aires: Aguilar.

Bourdieu, P. (1972). Esquise d'une theorie de la pratique. Geneve: Droz.

Bourdieu, P. (1996). Marginalia. Algumas notas adicionais sobre o dom. Mana, 2(2).

Caillé, A. (2002). Antropologia do Dom. O terceiro paradigma. Petropólis: Vozes.

Caillé, A. (1998). Nem holismo, nem individualismo metodológicos: Marcel Mauss e o paradigma da dádiva. Revista Brasileira de Ciências Sociais, 13(38).

Corcuff, P. (2011). As novas sociologias: construções da realidade social. Bauru, SP: EDUSC.

Damatta, R. (1979). Carnavais, malandros e heróis. Para uma sociologia do dilema brasileiro. Rio de Janeiro: Zahar.

Damo, A. S. (2007). Do dom à profissão. A formação de futebolistas no Brasil e na França. São Paulo: Aderaldo \& Rothschild Editores, Anpocs.

Davies, J. D. (2006). Profissionalização em projetos sociais: a experiência do Projeto Grael. Iniciação Científica. (Graduando em Bacharelado Em Ciências Sociais) - Universidade Federal Fluminense, Conselho Nacional de Desenvolvimento Científico e Tecnológico. Orientador: Simoni Lahud Guedes.

Decacche-Maia, E. (2003). Esporte e políticas públicas na virada do milênio: o caso de Niterói (Tese de Doutorado). Programa de Pós-Graduação em Antropologia Social, Museu Nacional, UFRJ.

Filmus, D. (org.) (1997). Elperfil de las ONGs en la Argentina. La Plata: Flacso, Banco Mundial.

Giulianotti, R. (2002). Sociologia do Futebol. Dimensões históricas e socioculturais do esporte das multidões. São Paulo: Nova Alexandria.

Godbout, J. (com Caillé, A.) (1999). O espírito da dádiva. Rio de Janeiro: Editora Fundação Getúlio Vargas.

Godelier, M. (2001). O enigma do dom. Rio de Janeiro: Civilização Brasileira.

Guedes, S. L. (1977). O futebol brasileiro: instituição zero (Dissertação Mestrado em Antropologia Social), Museu Nacional, UFRJ.

Guedes, S. L. (1997). Jogo de Corpo: um estudo de construção social de trabalhadores. Niterói: Eduff.

Guedes, S. L. (1998). O povo brasileiro no campo de futebol. O Brasil no campo de futebol. Estudos antropológicos sobre os significados do futebol brasileiro. Niterói: Eduff. 
Guedes, S. L. (1982). Subúrbio: celeiro de craques. En DaMatta (org.), Universo do futebol. Rio de Janeiro: Pinakotheke.

Guedes, S. L. (2003). Os europeus do futebol brasileiro ou como a pátria de chuteiras enfrenta a ameaça do mercado. Praia Vermelha (UFRJ), 8, 210-221.

Guedes, S. L., Davies, J., Novaes, R. (2006). Projetos sociais esportivos e as novas trajetórias dos atletas profissionais. $30^{\circ}$. Encontro Anual da ANPOCS.

Guedes, S. L. (2009). Control Social y el gran circuito del don. Proyectos pedagógicos deportivos en Argentina y Brasil. En Roberto Kant de Lima, Sofia Tiscornia, Lucia Eilbaum (org.), Burocracias penales, administración institucional de conflictos y ciudadanía. Experiencia comparada entre Brasil y Argentina (pp. 119-142). Buenos Aires: Antropofagia.

Guedes, S. L. (2014a). Los europeos del futbol brasileño o como la patria de chuteras enfrenta la amenaza do mercado. En Roldan et al. (org.). Naciones em campo: futbol, identidades y nacionalismios en America Latina (pp. 157-163). Bogotá: Kineisis.

Guedes, S. L. (2014b). A produção das diferenças na produção dos estilos de jogo no futebol: a propósito de um texto fundador. En Hollanda y Burlamaqui (org.), Desvendando o jogo. Nova luz sobre o futebol (pp. 153-172). Niterói: eduff.

Guedes, S. L. (2014c). A dádiva e os diálogos identitários através das copas do mundo no Brasil. En F. de Campos y D. Alfonsi. (org.), Futebol objeto das ciências humana. São Paulo: Leya.

Kant de Lima, R. (2008). Ensaios de antropologia e de direito. Rio de Janeiro: Editora Lumen Juris.

Lyra Filho, J. (1954). Taça do Mundo 1954. Rio de Janeiro: Pongetti.

Mauss, M. (2003). Ensaio sobre a dádiva. Em Sociologia e Antropologia. São Paulo: Cosac e Naify.

Melo, M. P. de (2005). Esporte e juventude pobre. Políticas públicas de lazer na Vila Olímpica da Maré. Campinas: Autores Associados.

Novaes, R. B. (2006). Esporte, profissionalização e estilos de vida: três experiências etnográfica. Trabalho de Conclusão de Curso. (Graduação em Bacharelado Em Ciências Sociais) - Universidade Federal Fluminense, Conselho Nacional de Desenvolvimento Científico e Tecnológico. Orientador: Simoni Lahud Guedes.

O’Donnel, G. (2004). Y a mí que mierda me importa? Notas sobre sociabilidad y politica en la Argentina y Brasil.En Contrapuntos. Ensayos escogidos sobre autoritarismo y democratización (pp. 165-193). Buenos Aires: Paidós.

Oliveira Filho, P. P. de A. (2005).É de menino que se torce o pepino. Concepções de masculinidade nos projetos sociais esportivos. Trabalho de Conclusão de Curso. (Graduação em Bacharelado Em Ciências Sociais) - Universidade Federal Fluminense, Conselho Nacional de Desenvolvimento Científico e Tecnológico. Orientador: Simoni Lahud Guedes.

Silva, R. C. (2007). Mapeamento de projetos sociais relacionados a personalidades esportivas famosas. Iniciação Científica. (Graduando em Bacharelado Em Ciências Sociais) - Universidade Federal Fluminense, Conselho Nacional de Desenvolvimento Científico e Tecnológico. Orientador: Simoni Lahud Guedes.

\section{Notas}

1 La disertación deberá publicarse este año, con un ensayo introductorio que evalúa los límites del trabajo entonces realizado y da cuenta de los desarrollos posteriores de las temáticas desarrolladas.

2 Río de Janeiro, en ese entonces, era la capital federal, siendo referencia cultural para todo el país.

3 Más tarde, reformé esta fecha: en realidad, el fenómeno al que me refiero se presenta desde 1938, con el desempeño del jugador Leônidas, conocido como Diamante Negro, en los campos franceses, en la III Copa del Mundo. Gilberto Freyre, en esa ocasión, clasifica al fútbol brasileño como "Football Mulato", en referencia a su tesis expresada en Casa Grande y Senzala, colaborando en esta interpretación (Guedes, 2014b).

4 Por ejemplo, estos obreros cuestionaban mucho la noción de la excepcionalidad de los cracks, argumentando que muchos de ellos eran también excelentes jugadores pero que no tuvieron "la chance y la oportunidad". (Guedes, 1977, 1982). 
5 Cf. http://peruzzolo.blogspot.com.br/2010/05/convocados-para-copa-do-mundo-de-1986.html, acceso el 6 de mayo de 2018

6 Cf. https://pt.wikipedia.org/wiki/Brasil_na_Copa_do_Mundo_FIFA_de_1990, acceso el 6 de mayo de 2018.

7 Cf. https://pt.wikipedia.org/wiki/Brasil_na_Copa_do_Mundo_FIFA_de_1994, acceso el 6 de mayo de 2018.

8 Cf. https://pt.wikipedia.org/wiki/Brasil_na_Copa_do_Mundo_FIFA_de_1998, acceso el 6 de mayo de 2018.

9 Por supuesto, hay una alternancia y una cierta imprecisión que no comprometen el argumento porque los números son bastante obvios.

10 Traducido al español del trabajo de la versión en portugués de Guedes.

11 Lo mismo ocurrió en Argentina: Lionel Messi, por ejemplo, como sabemos, salió directamente de las categorías inferiores de Newell's Old Boys para el Barcelona, no habiendo nunca jugado como profesional en Argentina.

12 En contraste, el mediocampista Zizinho, considerado el mejor jugador de la Copa de 1950, se habría convertido en fiscal de rentas (empleo burocrático de salario medio) tras su jubilación como jugador (véase https://es.wikipedia.org/wiki /Zizinho, acceso el 7 de mayo de 2018). Vivía en la ciudad de Niterói, donde murió. Habría dejado de herencia sólo un apartamento sencillo, de dos dormitorios, en una región no muy valorada de la ciudad, según algunos informes no verificables.

13 Estos epítetos se utilizaron hasta 2002 y no se utilizaron desde entonces.

14 Analicé este texto en Guedes, 1998.

15 Traducido al español del trabajo de la versión en portugués de Guedes.

16 Ya he publicado dos capítulos de libros analizando el material de esta investigación, bajo diferentes ángulos: en un caso, el control social (cf. Guedes, 2009) y en el otro, los diálogos de identidad (cf. Guedes, 2014c).

17 Con una excepción, que veremos más adelante.

18 Ensayo sobre el don. Formas y función del intercambio en las sociedades arcaicas (1968 [1923-1924])

19 Traducido al español del trabajo de la versión en portugués de Guedes.

20 Se utiliza a menudo la expresión de niños y jóvenes en riesgo social para designar a su objetivo. Sin embargo, como casi todos estos proyectos exigen que el niño esté matriculado en la escuela, excluyen un número grande de niños que no están escolarizados y para los cuales el proyecto social deportivo podría ser hasta más importante

21 Programa del Ministerio de Deportes a través del cual se ofrecen actividades deportivas y otras en el contraturno de las clases (Cf. http://portal.esporte.gov.br/snee/segundotempo/default.jsp, acceso el 9 de mayo de 2018).

22 El Instituto Ayrton Senna, Instituto Canhotinha de Ouro, Fundación Cafu, Fundación Romarinho, Instituto Fernanda Keller, Proyecto Grael, etc.

23 Este es el caso, por ejemplo, de varios atletas que colaboran con Zanetti en la fundación PUPI.

24 Obviamente, mantener su nombre en evidencia no es un objetivo menor, así como obtener descuentos en el impuesto sobre la renta. Sin embargo, quien conoce estos proyectos sociales sabe cuánto trabajo implica. Se trata, en realidad, de una forma muy compleja para obtener estas ganancias que podrían obtenerse de otras maneras mucho más simples.

25 Clasificada como "moderna" por tres razones: (1) no circula en las redes de parentesco y afinidad; (2) mantiene una relación "floja" con la religión; (3) no se funda en "una obligación moral de la clase acomodada hacia el pueblo" (p. 95), siendo encontrada en personas de cualquier medio social. Sin embargo, en el presente caso, como voy a sostener más adelante, hay otro tipo de obligación moral.

26 Traducido al español del trabajo de la versión en portugués de Guedes.

27 Recordemos la creación posterior de la Fundación Leonel Messi. 